\title{
Découverte d'une fausse contamination professionnelle par le tritium
}

\author{
G. AVENARD*, B. HERCELIN*, G. GILLIER**
}

RÉSUMÉ La surveillance des personnels exposés aux rayonnements ionisants dans les laboratoires de faible activité manipulant du tritium ou du ${ }^{14} \mathrm{C}$ est assurée par une analyse radiotoxicologique semestrielle des urines. Les auteurs rapportent, dans le cadre de cette surveillance systématique, une fausse contamination professionnelle dont ils discutent l'origine et le mécanisme. Bien que n'ayant pas manipulé de produits tritiés depuis plus de six mois, un animalier présente une excrétion urinaire de tritium (environ $500 \mathrm{~Bq} / 24 \mathrm{~h}$ ) faible mais persistante. Après plusieurs confirmations et une enquête approfondie au niveau du laboratoire, aucune explication n'est donnée. Compte tenu de la période biologique du tritium (10 $\mathrm{j}$ environ), il est décidé une hospitalisation afín de refaire un bilan complet en dehors du milieu professionnel ; c'est à ce moment que le port d'une montre en plastique, avec des aiguilles radioluminescentes, est soupçonné. Après abandon de sa montre, les contrôles s'avèreront négatifs et la montre ainsi que des montres du même type seront étudiées in vitro et in vivo chez l'animal de laboratoire (rat Hairless). Les montres en plastique sont susceptibles de laisser diffuser le tritium utilisé dans les peintures des cadrans ou des aiguilles radioluminescentes. La contamination se fait par absorption cutanée et/ou par inhalation et est environ 100 fois plus faible que la limite annuelle la plus basse pour les personnes exposées, donc sans danger pour la santé. II apparaît que les montres fabriquées depuis 1993 n'utilisent plus ce type de peintures ; mais compte tenu de la très grande diffusion de ces montres, il faut savoir penser à cette origine devant une excrétion de tritium faible mais persistante chez des personnels surveillés semestriellement.

ABSTRACT In laboratories using low amounts of radionuclides $\left({ }^{3} \mathrm{H}\right.$ or $\left.{ }^{14} \mathrm{C}\right)$, staff monitoring is carried out by urine analysis every six months. The authors report a false professional contamination and discuss the origin and the mechanism of absorption. Although a laboratory animal technician had not handled any ${ }^{3} \mathrm{H}-$ labelled product for at least six months, a check-up revealed $500 \mathrm{~Bq}$ tritium in the $24 \mathrm{~h}$ urine. This stable but very low radioactivity level persisted without explanation, despite an appropriate laboratory investigation. According to the tritium biological half-live (10 days), he was admitted to hospital for a complete check-up outside professional environment. We suspected that a watch with radioluminescent hands could be the problem. He left his watch and urine controls were negative. This watch and other watches of the same sort were investigated in vitro and in vivo in animals (hairless rats). Radioluminescent plastic case watches can release tritium, contamination occuring by cutaneous absorption and/or by inhalation. The intake is about $\mathbf{1 0 0}$ times lower than the annual limit

* Laboratoires Cassenne/Roussel UCLAF, 17 route de Pontoise, 95520 Osny.

** Service de médecine du travail, AMETIF, 7 avenue de la Palette, BP 58, 95020 Cergy Pontoise. 
of incorporation for non exposed people and has no health consequences. Tritium is no more used for this kind of watches, but on account of the large diffusion of plastic case watches, it seems that such contaminations could still be observed and interfere with urine monitoring of workers exposed to tritium.

\section{Introduction}

La surveillance médicale professionnelle des personnels exposés aux radionucléides est sous la responsabilité du Service central de protection contre les rayonnements ionisants $(\mathrm{SCPRI})^{1}$. Dans les laboratoires manipulant de très faibles activités, en particulier les émetteurs bêta de faible énergie $\left({ }^{3} \mathrm{H}\right.$ et $\left.{ }^{14} \mathrm{C}\right)$, et compte tenu du risque d'ingestion, de pénétration cutanée ou d'inhalation accidentelles, cette surveillance est réalisée par la radiotoxicologie des urines de $24 \mathrm{~h}[1,4]$. Les analyses sont pratiquées par le laboratoire d'analyses médicales du SCPRI et les résultats sont transmis au médecin du travail qui informe l'employé et l'employeur d'une éventuelle anomalie, afin de soustraire, le cas échéant, la personne de la liste des personnes aptes à la manipulation des radioéléments, et de réaliser une enquête sur l'origine d'une contamination accidentelle professionnelle.

Nous rapportons ici le cas de résultats positifs observés dans le cadre de cette surveillance professionnelle systématique et l'origine de la contamination est discutée.

\section{Observation}

M. L..., 39 ans, est amené en tant qu'animalier à manipuler des animaux ayant reçu des produits radiomarqués pour réaliser des études de pharmacocinétique de médicaments. A ce titre, il bénéficie d'une surveillance médicale systématique, notamment, sous la forme d'une analyse semestrielle sur les urines de $24 \mathrm{~h}$. En janvier 1993, le contrôle revient positif, montrant $480 \mathrm{~Bq}$ de tritium/24 h. M. L... n'ayant pas manipulé de produits tritiés depuis juin 1992 et le contrôle de juillet étant négatif, un second contrôle est demandé ainsi qu'une enquête sur le site afin de comprendre ce résultat. Le poste de travail de M. L... est momentanément modifié pour annuler le risque d'exposition aux radioéléments. Lors du contrôle de mars 1993, le résultat est toujours positif $(610 \mathrm{~Bq} / 24 \mathrm{~h})$. Compte tenu de la période biologique de 10 jours du tritium dans l'organisme, ce second résultat, un mois après le premier contrôle, ne pouvait s'expliquer par une contamination unique. L'enquête au niveau du laboratoire montrant le respect des procédures, l'absence de perte de sources, la vérification des aspects de sécurité et de sûreté, n'apportera aucun élément

1. Devenu l'Office de protection contre les rayonnements ionisants (OPRI). 
supplémentaire. Une auto-inspection sera même pratiquée et malgré 92 frottis réalisés afin de rechercher une contamination de surface, aucune explication ne sera donnée.

Deux contrôles supplémentaires seront réalisés chez M. L..., en mai et juin (700 et $960 \mathrm{~Bq} / 24 \mathrm{~h}$ ), montrant une persistance de cette excrétion urinaire de tritium ne pouvant être expliquée que par une contamination faible mais chronique ou accidentelle à l'occasion de chaque contrôle. Il sera donc proposé à M. L... d'être hospitalisé dans le service de médecine nucléaire de l'hôpital Avicenne, non pas en raison du niveau de la contamination qui était relativement bas, mais afin de réaliser des prélèvements sanguins et urinaires, en dehors du milieu professionnel. C'est devant la persistance de cette excrétion, à un niveau constant, que le SCPRI eut l'idée qu'une montre radioluminescente pouvait être en cause. M. L... abandonna donc sa montre et fut hospitalisé trois semaines plus tard; l'ensemble du bilan clinique et biologique se révéla tout à fait normal, aucune radioactivité ne fut décelée dans les urines lors de cette hospitalisation, ni lors d'un contrôle un mois plus tard.

La montre, in vitro, placée dans un incubateur sous vide à côté d'un bêcher de $10 \mathrm{ml}$ d'eau distillée laissa apparaître une fuite de tritium objectivée par comptage de l'eau du bécher : $17000 \mathrm{~Bq} / 72 \mathrm{~h}$. D'autres essais sur cette montre et des montres du même type ont été conduits afin de mieux comprendre le mode de contamination.

M. L... portait, en effet, nuit et jour depuis fin 1992, une montre en plastique avec des aiguilles radioluminescentes, se contaminant par des quantités minimes de tritium qui étaient retrouvées dans ses urines.

\section{Discussion}

Devant cette fausse contamination professionnelle due au port permanent d'une montre en plastique avec des aiguilles radioluminescentes, plusieurs questions se sont posées :

- Cette observation était-elle isolée ou méritait t-elle d'être connue de tous les médecins du travail en charge de radioprotection? Quel était le mécanisme de la fuite (limitée à cette montre ou se retrouvant sur d'autres modèles) et quel était le mode de contamination?

- Que représentait le niveau d'exposition observé par rapport au risque professionnel des personnes exposées?

Il est connu que les peintures radioluminescentes utilisées dans l'horlogerie sont préparées depuis de nombreuses années (après l'abandon du radium) à partir du tritium qui produit une photoluminescence au contact d'un sulfure de zinc $[2,8,9,11-12]$. Plusieurs auteurs décrivent la possibilité d'excrétion urinaire de tritium chez les travailleurs exposés (en particulier dans l'horlogerie) 
après contamination accidentelle ou lorsqu'il existe de mauvaises conditions de travail [8-9]. Par contre, l'observation d'excrétion urinaire de tritium chez des porteurs de montre semble plus rare et est moins souvent rapportée [4-5], d'une part du fait de la limitation de la surveillance radiotoxicologique des urines et vraisemblablement du fait que les montres en métal et en verre, étanches, sont imperméables aux liquides et aux gaz. Dans les deux publications citées, c'est à l'occasion de la surveillance d'une population dans son ensemble que quelques contaminations anecdotiques par des montres sont rapportées, sans précision sur le type des montres.

Ce n'est que récemment [3] qu'un auteur a étudié plus de 100 porteurs de montres en plastique et a observé une excrétion urinaire de tritium chez ceuxci variant de 20 à $1133 \mathrm{~Bq} / \mathrm{l}$, en rapport avec les habitudes de port de montre (de quelques heures par jour à un port permanent) ; ces observations confirmées par celle de M. L... [7] mettent en avant la perméabilité au gaz des boîtiers en plastique dans l'origine de ces contaminations récentes par le tritium.

Concernant le mécanisme de sortie du tritium à l'extérieur de la montre et le mode de contamination, nous avons réalisé quelques études in vitro et chez l'animal. In vitro, la montre de M. L..., ainsi que deux autres modèles de la même marque, pris au hasard, également avec des aiguilles ou un cadran radioluminescents relarguent du tritium lorsqu'elles sont simplement immergées dans un incubateur rempli d'eau à température ambiante et que l'on compte la radioactivité de l'eau après $24 \mathrm{~h}(4800 \mathrm{~Bq} / 24 \mathrm{~h})$. Il est probable que le passage se fait directement à travers le plastique de la montre sous forme de tritium $\left({ }^{3} \mathrm{H}\right)$ ou de vapeur d'eau tritiée $\left({ }^{3} \mathrm{H}_{2} \mathrm{O}\right)$, compte tenu de la bonne étanchéité à l'eau de ce type de montre.

Ces résultats paraissant stables et reproductibles, il nous a paru intéressant d'étudier par quelle voie l'animal se contaminait, voie cutanée et/ou aérienne par inhalation, ce fait étant décrit de façon contradictoire dans la littérature [8, 10]. Un rat hairless portant la montre autour de l'abdomen - le contact entre le boîtier et la peau était assuré par une compresse quotidiennement humidifiée présente une excrétion urinaire de tritium de l'ordre de $450 \mathrm{~Bq} / 24 \mathrm{~h}$ dès le $3^{\mathrm{e}}$ jour. Après ablation de la montre, la décroissance urinaire de tritium montre une période biologique de l'ordre de $3,5 \mathrm{j}$ et une persistance de l'excrétion urinaire après $10 \mathrm{j}(70 \mathrm{~Bq} / 24 \mathrm{~h})$, confirmant ainsi les données publiées [10] sur l'absorption cutanée chez le rat. Deux autres rats ont été placés dans des cages contiguës, mais séparées de telle façon qu'ils respirent dans un espace confiné ; l'un (rat 1) porte la montre comme dans l'expérience précédente et.l'autre est libre (rat 2) dans la cage contiguë. Le rat 2 excrète environ $50 \%$ de la quantité de tritium excrétée par le rat 1 , mettant ainsi en évidence, dans ces conditions de proximité, le rôle de l'inhalation dans la contamination. Afin de conforter ces résultats, la montre a été attachée à la grille d'une cage dans laquelle ont été placés deux rats "normaux"; tous deux se contaminant par inhalation sans contact direct avec la montre, mais dans une proportion plus faible que lorsque la montre, correctement humidifiée, est en contact direct avec la peau. 
Au vu de ces résultats, il apparaît donc probable que, chez l'homme, le passage cutané joue un rôle prépondérant, en particulier chez les sujets portant la montre de façon permanente, se lavant les mains fréquemment ou manipulant beaucoup d'eau dans la journée, ce qui était le cas -de M. L.... Néanmoins, la contamination par inhalation reste possible avec ce type de montre et doit être gardée à l'esprit, en particulier lors de la pose sur une table de nuit, à proximité de la tête, dans une chambre peu ventilée.

Si l'on rapproche les valeurs d'excrétion urinaire trouvées dans notre observation (environ $500 \mathrm{~Bq} / 24 \mathrm{~h}$ ), de la valeur trouvée in vitro sur les montres (environ $5000 \mathrm{~Bq} / 24 \mathrm{~h}$ ), il faut admettre une incorporation d'environ $1 / 10^{\mathrm{e}}$ de la radioactivité apparente relarguée par la montre. Les limites admissibles, pour les travailleurs exposés, sont fixées par la Commission internationale de protection radiologique (CIPR). La limite annuelle d'incorporation (LAI) pour le tritium varie de $310^{9} \mathrm{~Bq}$ à $0,310^{9} \mathrm{~Bq} /$ an en fonction de la catégorie de personnel. Si l'on prend comme référence la valeur la plus basse, correspondant au public, la quantité de tritium sortant de la montre en un an est de $5000 \mathrm{~Bq} \times$ 365 jours, soit $210^{6} \mathrm{~Bq} / \mathrm{an}$, ce qui correspond à moins de $1 / 100^{e}$ de la LAI la plus basse.

Il s'agit donc d'une valeur très faible, d'un émetteur bêta de faible énergie qui ne présente aucun risque d'accumulation dans l'organisme compte tenu de sa période biologique courte ( $10 \mathrm{j}$ environ). Une telle activité est donc sans danger pour la santé des porteurs de montres. Néanmoins, ces contaminations paraissent d'autant plus paradoxales que le tritium avait l'avantage d'être un émetteur bêta de faible énergie dont le rayonnement ne pouvait traverser le métal ou le verre des montres et qu'un passage direct du gaz ou indirectement sous forme de vapeur d'eau à travers le plastique n'avait, semble-t-il, pas été envisagé par les fabricants. En effet, malgré le risque médical négligeable, mais afin de satisfaire au principe d'une exposition la plus minime possible aux rayonnements ionisants, nous avons informé le fabricant de montres. Celui-ci nous a précisé qu'il n'utilisait plus de tritium dans la fabrication des montres depuis le premier trimestre 1993, ayant été prévenu par un laboratoire allemand dans les mêmes circonstances que cette observation.

En conclusion, malgré l'arrêt récent de l'utilisation de tritium par l'un des fabricants de montres en plastique, ce type d'observation mérite d'être connu des médecins du travail qui risquent d'être confrontés à de telles fausses contaminations professionnelles pendant plusieurs années encore, compte tenu du nombre de ces montres, très à la mode, vendues depuis quelques années et de la période de 10 ans du tritium.

\section{Remerciements}

Nous remercions vivement J. Remenieras et M.L. Rémy (LABM de l'OPRI) pour l'aide apportée dans le cadre de cette observation et J.L. Moretti (Service de médecine nucléaire, Hôpital Avicenne) pour avoir organisé les hospitalisations. 


\section{RÉFÉRENCES}

[1] AUBOIROUX B. - La radioprotection dans les laboratoires utilisateurs de radioéléments. L'Actualité chimique, 1993, octobre/novembre, 62-66.

[2] BRADLEY F.J., BLAIS R., JONES A. - Impact of tritium on the watch industry, 1966-1970. Radiol. Health Data Rep., 1971, 601-610.

[3] BRUNNER P., SCHNEIDER P., SCHEICHER H., SEYERL G., KURNIK P., ENNEMOSER O., AMBACH W. - Tritium exposure from plastic case watches. Lancet (letter), 1994, $n^{\circ} 343,116$.

[4] DUISJINGS J.H., BEENTJES L.B., COOPS A.J., VAN DER JAGT P.J. - Internal contamination of radiological workers by tritium compounds at two universities in The Netherlands. Health Phys., 1984, 46, 665-669.

[5] FITZSIMMONS C.K., McNELIS D.N., WRUBLE D.T. - Tritium activity in urine from luminous dial wrist watches. Health Phys., 1972, 22, 514-516.

[6] FRANCE. Décret $n^{\circ} 86-1103$ du 2 octobre 1986 relatif à la protection des travailleurs contre les dangers des rayonnements ionisants. J.O.R.F., 12 octobre 1986. In : Protection contre les rayonnements ionisants (Brochure $n^{\circ} 1420$ ). Paris : Journal officiel, 1990, vol. 2, 427-453.

[7] GILLIER D., HERCELIN B., AVENARD G., MORETTI J.L., REMY M.L., REMENIERAS J. - Plastic watches and urinary excretion of tritium. Lancet (letter), 1994, $n^{\circ} 343,669-670$.

[8] LAMBERT B.E., VENNART J. - Radiation doses received by workers using tritium in industry. Health Phys., 1972, 22, 23-30.

[9] MOGHISSI A.A., TOERBER E.D., REGNIER J.E., CARTER N.W. and POSEY C.D. - Health physics aspects of tritium luminous dial painting. Health Phys., 1970, 18, 255-261.

[10] REHNBERG B.F., MOGHISSI A.A., WHEELER J.K., COLVIN M.C. - Percutaneous absorption by rats of tritium from a luminous compound. Health Phys., 1972, 22, 31-34.

[11] VENNART J. - Radiological protection in the luminising industry. Health Phys., 1970, 19, 835-836.

[12] WHEELER J.K., MOGHISSI A.A., REHNBERG B.F., COLVIN M.C. - Comparison between the biological half-life of a tritiated luminous compound with that of tritiated water in rats and cats. Health Phys., 1972, 22, 35-38. 